Higher-order moments and overlaps of Cartesian beams

This article has been downloaded from IOPscience. Please scroll down to see the full text article.

2010 J. Opt. 12065702

(http://iopscience.iop.org/2040-8986/12/6/065702)

View the table of contents for this issue, or go to the journal homepage for more

Download details:

IP Address: 131.215.220.165

The article was downloaded on 02/08/2010 at 17:27

Please note that terms and conditions apply. 


\title{
Higher-order moments and overlaps of Cartesian beams
}

\author{
Miguel A Bandres ${ }^{1}$, Dorilian Lopez-Mago ${ }^{2}$ and \\ Julio C Gutiérrez-Vega ${ }^{2}$ \\ ${ }^{1}$ California Institute of Technology, Pasadena, CA 91125, USA \\ ${ }^{2}$ Photonics and Mathematical Optics Group, Tecnológico de Monterrey, Monterrey, 64849, \\ Mexico
}

Received 4 February 2010, accepted for publication 19 April 2010

Published 28 May 2010

Online at stacks.iop.org/JOpt/12/065702

\begin{abstract}
We introduce a closed-form expression for the overlap between two different Cartesian beams. In the course of obtaining this expression, we establish a linear relation between the overlap of circular beams with azimuthal symmetry and the overlap of Cartesian beams such that the knowledge of the former allows the latter to be calculated very easily. Our formalism can be easily applied to calculate relevant beam parameters such as the normalization constants, the $M^{2}$ factors, the kurtosis parameters, the expansion coefficients of Cartesian beams, and therefore of all their relevant special cases, including the standard, elegant, and generalized Hermite-Gaussian beams, cosh-Gaussian beams, Lorentz beams, and Airy beams, among others.
\end{abstract}

Keywords: Cartesian beams, intensity moments, $M^{2}$ factor, kurtosis, Appell, hypergeometric functions

\section{Introduction}

Finding explicit expressions for the overlap integrals and higher-order moments of optical beams is of importance to characterize and analyze the behavior of the beams from a theoretical and also a practical point of view. In a recent paper [1], we introduced a very general and closed-form expression for the overlap and moments between two different beams with azimuthal symmetry, i.e. circular beams [2]. This expression was used to calculate relevant beam parameters such as the higher-order moments, the normalizations, the $M^{2}$ factors, the kurtosis parameters, and the expansion coefficients between all the special cases of circular beams.

On the other hand, Cartesian beams (CB) were introduced by Bandres and Gutiérrez-Vega as the general solution of the paraxial wave equation in Cartesian coordinates [3]. Besides the possibility of obtaining novel and meaningful beam structures, one of the most important aspects of Cartesian beams is that, for special values of their parameters, they reduce to known families of optical beams including the standard, elegant, and generalized Hermite-Gaussian beams [4], cosine-Gaussian and cosh-Gaussian beams [5], Lorentz beams [6, 7], fractional-order elegant HermiteGaussian beams [8], and Airy beams [9, 10]. Finding general properties of Cartesian beams is a way to characterize all these special cases at the same time.

In this paper, we establish a relation between the overlap of the circular beams [2] and the overlap of Cartesian beams [3] such the knowledge of the former allows to calculate very easily the latter. We apply this connection to determine the explicit expression for the $2 v$-order overlap

$$
\sigma_{1,2}^{(2 v)}=\int_{-\infty}^{\infty} x^{2 v} U_{1}(x) U_{2}^{*}(x) \mathrm{d} x,
$$

between two Cartesian beams $U_{1}(x)$ and $U_{2}(x)$ with arbitrary parameters. Finding $\sigma_{1,2}^{(2 v)}$ contributes to the general characterization of Cartesian beams, and also provides an unified expression for calculating relevant beam parameters such as the higher-order moments, the normalizations, the $M^{2}$ factors, the kurtosis parameters, and the expansion coefficients between all the relevant special cases mentioned above. We remark that the expressions for the overlaps and intensity moments are numerically valid even for complex values of the order $2 v$. However, since physical meaning and applications of the overlaps and moments with real integer values of $2 v$ are important and well known in beam optics, in this paper we restrict ourselves to discussing in detail this situation for Cartesian beams. Definitely, the possibility of having 
analytical expressions for overlaps with fractional and even complex orders, allows one to extend the analysis and give new physical interpretations for the overlaps of Cartesian beams.

\section{Definition of a Cartesian beam}

The transverse distribution at the output plane of a paraxial ABCD system of even and odd Cartesian beams is described by the confluent hypergeometric function ${ }_{1} F_{1}(a, b ; x)$ as follows [3]:

$$
{ }_{t} U_{\beta}(x ; q, p)=\zeta_{t}\left(P x^{2}\right)^{(t-1 / 2) / 2}{ }_{1} F_{1}\left(\beta, t ; P x^{2}\right) \exp \left(\frac{\mathrm{i} k x^{2}}{2 q}\right),
$$

where $t$ is the parity parameter defined by

$$
t \equiv \begin{cases}1 / 2, & \text { for even (e) beams } \\ 3 / 2, & \text { for odd (o) beams }\end{cases}
$$

$k$ is the wavenumber, $\beta \in \mathbb{C}$ is a complex parameter, and

$$
P=P(q, p) \equiv \frac{\mathrm{i} k}{2}\left(\frac{1}{p}-\frac{1}{q}\right) \text {. }
$$

Cartesian beams are characterized by two complex beam parameters $(q, p)$ whose values at the output plane of the system are related to their values $\left(q_{0}, p_{0}\right)$ at the input plane $(z=0)$ by the usual bilinear transformations

$$
q=\frac{A q_{0}+B}{C q_{0}+D}, \quad p=\frac{A p_{0}+B}{C p_{0}+D} .
$$

In (2), the factor

$$
\zeta_{t} \equiv \frac{\left(A+B / q_{0}\right)^{\beta-(t+1 / 2) / 2}}{\left(A+B / p_{0}\right)^{\beta-(t-1 / 2) / 2}}
$$

is an overall amplitude factor arising from the propagation of the beam through the ABCD system. In general, the fulfilment of the conditions

$$
\operatorname{Im}\left(1 / q_{0}\right)>0, \quad \operatorname{Im}\left(1 / p_{0}\right)>0,
$$

ensures the finiteness of the beam power across the whole transverse plane [2]. The second condition in (7) is not necessary for the special case of generalized HermiteGaussian beams for which $\operatorname{Im}\left(1 / p_{0}\right)$ can take any value.

The free space propagation of Cartesian beams along a distance $z$ can be directly obtained from (2)-(6) by replacing the values $[A, B ; C, D]=[1, z ; 0,1]$. Equation (2) reduces to known families of optical beams with rectangular symmetry for some special values of the parameters $\left(\beta, q_{0}, p_{0}\right)$. For reference purposes, in the appendix we include a table of these special cases and the explicit expressions for their propagations and quality factors $M^{2}$. It should be mentioned that Cartesian beams were also discussed by Torre $[11,12]$ employing a Lie algebra formalism.

\section{Overlap and $2 v$-order intensity moments of Cartesian beams}

To evaluate the overlap integral (1) for Cartesian beams (2) consider the transverse field of two different beams at the output plane of an ABCD system, i.e.

$$
{ }_{t_{1}} U_{1}={ }_{t_{1}} U_{\beta_{1}}\left(x ; q_{1}, p_{1}\right), \quad{ }_{t_{2}} U_{2}={ }_{t_{2}} U_{\beta_{2}}\left(x ; q_{2}, p_{2}\right),
$$

where $\left(q_{j}, p_{j}\right), j=\{1,2\}$, are given by (5).

Due to the even and odd parities of Cartesian beams, it is convenient to consider separately the following cases:

(i) Overlap between beams with the same parity, $t_{1}=t_{2}=t$, i.e.

$$
\begin{aligned}
{ }_{t} \sigma_{1,2}^{(2 v)} & =\int_{-\infty}^{\infty} x^{2 v}{ }_{t} U_{1}{ }_{t} U_{2}^{*} \mathrm{~d} x \\
& =\left[1+(-1)^{2 v}\right] \int_{0}^{\infty} x^{2 v}{ }_{t} U_{1{ }_{t}} U_{2}^{*} \mathrm{~d} x .
\end{aligned}
$$

This relation holds for complex values of $v$, but note that ${ }_{t} \sigma_{1,2}^{(2 v)}$ vanishes for $2 v=1,3,5, \ldots$.

(ii) Overlap between beams with opposite parity, $t_{1}=1 / 2$, $t_{2}=3 / 2$, i.e.

$$
\begin{aligned}
e_{o} \sigma_{1,2}^{(2 v)} & =\int_{-\infty}^{\infty} x^{2 v}{ }_{e} U_{1 o} U_{2}^{*} \mathrm{~d} x \\
& =\left[1-(-1)^{2 v}\right] \int_{0}^{\infty} x^{2 v}{ }_{e} U_{1 o} U_{2}^{*} \mathrm{~d} x .
\end{aligned}
$$

This relation holds for complex values of $v$, but note that $e_{e} \sigma_{1,2}^{(2 v)}$ vanishes for $2 v=0,2,4, \ldots$

\subsection{Overlap between Cartesian beams with the same parity and its relation with the overlap of Circular beams}

Let us first concentrate our attention on the overlap between beams with the same parity. Actually, this is the most important situation because it encompasses, as particular cases, the higher-order intensity moments of Cartesian beams.

By substituting the expressions (2) into (9b) and comparing the resulting integral with (8)-(9) of [1] we find that the overlap ${ }_{t} \sigma_{1,2}^{(2 v)}$ of the Cartesian beams ${ }_{t} U_{\beta}(x ; q, p)$ is proportional to the overlap $\mathcal{Q}_{1,2}^{(2 v)}$ of the Circular beams $U_{\beta}^{m}(r, \theta ; q, p)$ according to the following relation:

$$
{ }_{t} \sigma_{1,2}^{(2 v)}=\left.\boldsymbol{a} \mathcal{Q}_{1,2}^{(2 v)}\right|_{m=t-1},
$$

where

$$
\boldsymbol{a} \equiv \frac{1}{2 \pi}\left[1+(-1)^{2 v}\right]\left(P_{01} P_{02}^{*}\right)^{1 / 4},
$$

is a proportionality constant, $P_{01}, P_{02}$ are the values of the beam parameters $P_{1}, P_{2}$ at $z=0$, and the overlap $\mathcal{Q}_{1,2}^{(2 \nu)}$ of the Circular beams evaluated at $m=t-1$ is given by [1]

$$
\begin{gathered}
\left.\mathcal{Q}_{1,2}^{(2 v)}\right|_{m=t-1}=\pi \Gamma(v+t) \zeta_{1} \zeta_{2}^{*} \frac{\left(P_{1} P_{2}^{*}\right)^{(t-1) / 2}}{\left(S_{12}\right)^{v+t}} \\
\quad \times F_{2}\left(v+t ; \beta_{1}, \beta_{2}^{*} ; t, t ; \frac{P_{1}}{S_{12}}, \frac{P_{2}^{*}}{S_{12}}\right),
\end{gathered}
$$

with $F_{2}\left(a ; b, b^{\prime} ; c, c^{\prime} ; x, y\right)$ denoting the $F_{2}$ Appell hypergeometric function $[1,13,14]$ and $S_{12} \equiv \mathrm{i} k\left(1 / q_{2}^{*}-1 / q_{1}\right) / 2$. 
The $2 v$-order intensity moment of the Cartesian beams is obtained by setting $U_{1}=U_{2}=U_{\beta}$ in (11), we have

$$
{ }_{t} \sigma^{(2 v)}=\left.\boldsymbol{b} \mathcal{Q}^{(2 v)}\right|_{m=t-1},
$$

where

$$
\boldsymbol{b} \equiv \frac{1}{2 \pi}\left[1+(-1)^{2 v}\right]\left|P_{0}\right|^{1 / 2},
$$

is the proportionality constant, $\mathcal{Q}^{(2 v)}$ is the $2 v$-order intensity moment of the Circular beams [1]

$$
\begin{aligned}
& \left.\mathcal{Q}^{(2 v)}\right|_{m=t-1}=\frac{\pi \Gamma(v+t)\left|\zeta_{t}\right|^{2}|P|^{t-1}}{S^{v+t}} \\
& \quad \times F_{2}\left(v+t ; \beta, \beta^{*} ; t, t ; \frac{P}{S}, \frac{P^{*}}{S}\right),
\end{aligned}
$$

and

$$
S \equiv k \operatorname{Im}(1 / q),
$$

is a positive real quantity.

Equations (11) and (14) constitute an important and useful result of this paper. Because the overlaps of the Cartesian and Circular beams are proportional to each other, all the results reported in [1] for the Circular beams can be straightforwardly applied to the case of the Cartesian beams. Furthermore, because the proportionality constants $\boldsymbol{a}$ and $\boldsymbol{b}$ do not depend on the propagation distance, the behavior of the overlaps and higher-order moments of the Cartesian beams upon propagation is exactly the same as that of the Circular beams. In what follows, since all results can be easily derived from [1], we will omit the details of the derivation and give only the most important results for the Cartesian beams.

The beam power ${ }_{t} \sigma^{(0)}$ of the Cartesian beams corresponds to the zeroth-order intensity moment and is calculated by setting $v=0$ in (14). The identity (9.182.3) of [14] allows us to rewrite ${ }_{t} \sigma^{(0)}$ in terms of the conventional Gauss hypergeometric function $F={ }_{2} F_{1}(a, b ; c ; x)$ as follows:

$$
{ }_{t} \sigma^{(0)}=\left|\frac{\Gamma(t) \bar{a}}{\bar{c}^{2 \beta} \bar{b}^{1 / 2-t}}\right| F\left(\beta, \beta^{*} ; t ; \bar{w}\right),
$$

where the overlined quantities

$$
\begin{gathered}
\bar{a} \equiv\left|\frac{1}{\left(A+B / q_{0}\right) \sqrt{S}}\right|=\frac{1}{\sqrt{S_{0}}}, \\
\bar{b}=\left|\frac{\left(A+B / p_{0}\right)}{\left(A+B / q_{0}\right)} \frac{P}{S}\right|=\left|\frac{P_{0}}{S_{0}}\right|, \\
\bar{c} \equiv\left(1-\frac{P}{S}\right) \frac{A+B / p_{0}}{A+B / q_{0}}=1-\frac{P_{0}}{S_{0}}, \\
\bar{w} \equiv\left|\frac{P}{S-P}\right|^{2}=\left|\frac{P_{0}}{S_{0}-P_{0}}\right|^{2},
\end{gathered}
$$

represent quantities whose values remain invariant under propagation through paraxial systems characterized by unimodular $\mathrm{ABCD}$ matrices (i.e. $A D-B C=1$ ) with real elements.
As occurs for the Circular beams [1], the $2 n$-order moment of the Cartesian beams can also be evaluated using the recursive relation

$$
\begin{aligned}
{ }_{t} \sigma^{(2 n)} & =-\frac{\mathrm{d}}{\mathrm{d} S}{ }_{t} \sigma^{(2 n-2)}=\left(-\frac{\mathrm{d}}{\mathrm{d} S}\right)^{n}{ }_{t} \sigma^{(0)}, \\
n & =1,2,3, \ldots,
\end{aligned}
$$

which is valid for both even and odd parities. For example, by differentiating ${ }_{t} \sigma^{(0)}(18)$ with respect to $-S$ we obtain the following expressions for the second-order intensity moments of the Cartesian beams.

(i) Beams with even parity $(t=1 / 2)$

$$
e_{e} \sigma_{\beta}^{(2)}=-\frac{\mathrm{d}_{e} \sigma_{\beta}^{(0)}}{\mathrm{d} S}={ }_{e} G_{\beta} e \sigma_{\beta}^{(0)}+{ }_{e} H_{\beta}{ }_{o} \sigma_{\beta+1}^{(0)},
$$

where ${ }_{e} \sigma_{\beta}^{(0)}$ and ${ }_{o} \sigma_{\beta+1}^{(0)}$ are the powers of the beams ${ }_{e} U_{\beta}$ and ${ }_{o} U_{\beta+1}$, respectively, and the factors ${ }_{e} G_{\beta}$ and ${ }_{e} H_{\beta}$ depend on the beam parameters upon

$$
\begin{gathered}
{ }_{e} G_{\beta} \equiv \operatorname{Re}\left[\frac{2 \beta}{S}\left(\frac{P}{S-P}\right)+\frac{1}{2 S}\right], \\
{ }_{e} H_{\beta} \equiv \operatorname{Re}\left[8|\beta|^{2} \frac{|P|}{S(S-P)}\left|\frac{A+B / p_{0}}{A+B / q_{0}}\right|\right] .
\end{gathered}
$$

(ii) Beams with odd parity $(t=3 / 2)$

$$
{ }_{o} \sigma_{\beta}^{(2)}=-\frac{\mathrm{d}_{o} \sigma_{\beta}^{(0)}}{\mathrm{d} S}={ }_{o} G_{\beta}{ }_{o} \sigma_{\beta}^{(0)}+{ }_{o} H_{\beta e} \sigma_{\beta+1}^{(0)}+{ }_{o} E_{\beta}{ }_{o} \sigma_{\beta+1}^{(0)},
$$

where ${ }_{o} \sigma_{\beta}^{(0)},{ }_{e} \sigma_{\beta+1}^{(0)}$ and ${ }_{o} \sigma_{\beta+1}^{(0)}$ are the powers of the beams ${ }_{o} U_{\beta},{ }_{e} U_{\beta+1}$, and ${ }_{o} U_{\beta+1}$ respectively, and the factors ${ }_{o} G_{\beta}$, ${ }_{o} H_{\beta}$ and ${ }_{o} E_{\beta}$ depend on the beam parameters as

$$
\begin{gathered}
{ }_{o} G_{\beta} \equiv \operatorname{Re}\left[\frac{3}{2 S}+\frac{2 \beta P}{S^{2} \bar{c}}\left(\frac{A+B / p_{0}}{A+B / q_{0}}\right)\right], \\
{ }_{o} H_{\beta} \equiv \operatorname{Re}\left[-\left|\frac{\beta}{1 / 2-\beta}\right|^{2} \frac{\left|\bar{c}^{2}\right|}{S-P} \frac{(\bar{w}-1) \bar{b}}{2}\right], \\
{ }_{o} E_{\beta} \equiv \operatorname{Re}\left[-\left|\frac{\beta}{1 / 2-\beta}\right|^{2} \frac{\left|\bar{c}^{2}\right|}{S-P}(1+4 \bar{w} \operatorname{Re} \beta)\right] .
\end{gathered}
$$

\subsection{Fourier transform of a Cartesian beam and its $2 v$-order} moment ${ }_{t} \tilde{\sigma}^{2 v}$

We now determine the $2 v$-order moment of the power spectrum of the Cartesian beam. Let $k_{x}$ be the transverse position in Fourier space. The one-dimensional Fourier transform

$$
\tilde{U}\left(k_{x}\right)=\frac{1}{\sqrt{2 \pi}} \int_{-\infty}^{\infty} U(x) \exp \left(-\mathrm{i} k_{x} x\right) \mathrm{d} x
$$

can be obtained by propagating the beam $U(x)$ through a classical $2 f$ optical system characterized by the ABCD matrix $[0, k ;-1 / k, 0]$. Replacing the corresponding values into (2)(6) we get

$$
{ }_{t} \tilde{U}_{\beta}\left(k_{x}\right)=\left(\mathrm{i} / k_{x}\right){ }_{t} U_{\beta}\left(k_{x} ; \tilde{q}, \tilde{P} ; \tilde{\zeta}_{t}\right),
$$


where

$$
\tilde{q} \equiv-\frac{k^{2}}{q}, \quad \tilde{p} \equiv-\frac{k^{2}}{p}, \quad \tilde{P} \equiv \frac{\mathrm{i}(q-p)}{2 k},
$$

and

$$
\tilde{\zeta}_{t} \equiv \frac{\left(C+D / q_{0}\right)^{\beta-(t+1 / 2) / 2}}{\left(C+D / p_{0}\right)^{\beta-(t-1 / 2) / 2}} .
$$

Because $U(x)$ and $\tilde{U}\left(k_{x}\right)$ have the same mathematical structure, (14) can be directly applied to find the propagation of the $2 \nu$-order moment $\tilde{\sigma}^{(2 v)}$ of the power spectrum through ABCD systems, we obtain

$$
{ }_{t} \tilde{\sigma}^{(2 v)}={ }_{t} \sigma^{(2 v)}(\tilde{P}, \tilde{S}, \tilde{\zeta}) / k^{2}, \quad \tilde{S} \equiv-\operatorname{Im}(q) / k .
$$

\subsection{Overlap between beams with different parity}

Unlike the overlap between Cartesian beams with the same parity, the overlap between beams with different parity (10a) cannot be expressed in terms of the overlap of the Circular beams. To determine it, we start by replacing the beam expressions (2) into (10a), we then make the change of variables $t=r^{2}$, and apply (2.2) of [13] to get the following explicit expression

$$
\begin{gathered}
e_{e o} \sigma_{1,2}^{(2 v)}=\left[\frac{1-(-1)^{2 v}}{2}\right] \frac{e \zeta_{1 o} \zeta_{2}^{*}}{S_{12}^{1+v}} \sqrt{P_{2}^{*}} \Gamma(v+1) \\
\times F_{2}\left(v+1 ; \beta_{1}, \beta_{2}^{*} ; \frac{1}{2}, \frac{3}{2} ; \frac{P_{1}}{S_{12}}, \frac{P_{2}^{*}}{S_{12}}\right),
\end{gathered}
$$

for $\operatorname{Re}(\nu+1)>0$. Note that (33) vanishes for $2 \nu=0,2,4, \ldots$

\section{ABCD formulation for higher-order moments of general operators}

As happens for the Circular beams [1], also in the case of Cartesian beams it is possible to establish a general $\mathrm{ABCD}$ formulation of higher-order moments employing a general operator formalism. The propagation of moments of the Wigner distribution function through first-order optical systems has been studied by several authors within the context of the Lie algebra based approach to optics [15-21]. In particular, the propagation laws for the second- and fourthorder moments follow as particular cases of the general transfer law, proposed by Onciul [17] for moments up to fourth-order and later generalized to higher-order moments by Dragoman [18]. In this section we include the explicit expressions for the second- and fourth-order moments of Cartesian beams in terms of the beam parameters at a given initial plane of an ABCD system. These moments can be propagated through the systems by applying the rules studied in [18].

\subsection{Definition of the second-and fourth-order operators and moments}

Let $\hat{\mathbf{x}}=x$ and $\hat{\mathbf{p}}_{x}=(1 / \mathrm{i} k) \partial / \partial x$ be the transverse position and momentum operators. The following second-order operators are defined in terms of $\hat{\mathbf{x}}$ and $\hat{\mathbf{p}}_{x}$,

$$
\hat{\rho} \equiv \hat{\mathbf{x}} \cdot \hat{\mathbf{x}}=x^{2},
$$

$$
\begin{gathered}
\hat{\tau} \equiv \frac{\hat{\mathbf{x}} \cdot \hat{\mathbf{p}}_{x}+\hat{\mathbf{p}}_{x} \cdot \hat{\mathbf{x}}}{2}=\frac{1}{\mathrm{i} 2 k}\left(x \frac{\partial}{\partial x}+\frac{\partial}{\partial x} x\right), \\
\hat{\kappa} \equiv \hat{\mathbf{p}}_{x} \cdot \hat{\mathbf{p}}_{x}=-\frac{1}{k^{2}} \frac{\partial^{2}}{\partial x^{2}}
\end{gathered}
$$

where the commutation relations for $\hat{\rho}, \hat{\tau}$, and $\hat{\kappa}$ hold

$$
\begin{aligned}
{[\hat{\rho}, \hat{\tau}] } & =(\mathrm{i} 2 / k) \hat{\rho}, \\
{[\hat{\kappa}, \hat{\tau}] } & =-(\mathrm{i} 2 / k) \hat{\kappa}, \\
{[\hat{\kappa}, \hat{\rho}] } & =-(\mathrm{i} 4 / k) \hat{\tau} .
\end{aligned}
$$

Let

$$
\langle\hat{\alpha}\rangle=\frac{1}{\sigma^{(0)}} \int_{-\infty}^{\infty} U^{*}(\hat{\alpha} U) d x,
$$

be the normalized expectation value of the operator $\hat{\alpha}$ taken with respect to the function $U(x)$, where $\sigma^{(0)}=\int_{-\infty}^{\infty}|U|^{2} \mathrm{~d} x$ is the power of the beam $U(x)$.

The normalized second-order moments $\langle\hat{\rho}\rangle,\langle\hat{\tau}\rangle$, and $\langle\hat{\kappa}\rangle$ of the Cartesian beam can be physically interpreted as follows:

(i) $\langle\hat{\rho}\rangle=\sigma^{(2)} / \sigma^{(0)}$ is the normalized second-order intensity moment of the beam.

(ii) $\langle\hat{\tau}\rangle$ takes an integral representation $\langle\hat{\tau}\rangle=\left(1 / \sigma^{(0)}\right) \int_{-\infty}^{\infty}$ $x p_{x} \mathrm{~d} x$ with $p_{x}(x)=\operatorname{Im}\left[U^{*}(\mathrm{~d} U / \mathrm{d} x)\right]$ being the transverse component of the momentum density. The value of $\langle\hat{\tau}\rangle$ is related to the net momentum flowing in the transverse direction at the observation plane. The beam waist corresponds to the condition $\langle\hat{\tau}\rangle=0$. Positive (negative) values of $\langle\hat{\tau}\rangle$ mean that the beam is diverging (converging) as it propagates in the positive $z$ direction.

(iii) $\langle\hat{\kappa}\rangle k^{2}=\tilde{\sigma}^{(2)} / \sigma^{(0)}$ is the normalized second-order moment of the Fourier transform $\tilde{U}\left(k_{x}\right)$ of the beam, i.e. $\tilde{\sigma}^{(2)}=$ $\int_{-\infty}^{\infty} k_{x}^{2}|\tilde{U}|^{2} \mathrm{~d} k_{x}$.

The explicit expressions for the values $\langle\hat{\rho}\rangle_{0},\langle\hat{\tau}\rangle_{0}$, and $\langle\hat{\kappa}\rangle_{0}$ at the input plane in terms of the parameters of the Cartesian beam can be straightforwardly obtained from (33) and (34) of [1] by making the replacement $m \rightarrow t-1$, namely

$$
\begin{aligned}
& {\left[\begin{array}{c}
\langle\hat{\rho}\rangle_{0} \\
\langle\hat{\tau}\rangle_{0} \\
\langle\hat{\kappa}\rangle_{0}
\end{array}\right]=\operatorname{Re}\left\{\left[\begin{array}{ccc}
1 & 1 & 1 \\
1 / q_{0}^{*} & 1 / q_{0}^{*} & \left(1 / q_{0}+1 / p_{0}^{*}\right) / 2 \\
\left|1 / q_{0}\right|^{2} & 1 / q_{0}^{* 2} & 1 / q_{0} p_{0}^{*}
\end{array}\right]\right.} \\
& \left.\quad \times\left[\begin{array}{c}
J \\
K \\
L
\end{array}\right]\right\}
\end{aligned}
$$

where

$$
\begin{gathered}
J \equiv \frac{t}{S_{0}} \\
K \equiv \frac{2 \beta P_{0}}{S_{0}\left(S_{0}-P_{0}\right)}, \\
L \equiv \frac{\beta}{t^{2}} \frac{P_{0} K^{*}}{\left|P_{0}\right|} \frac{\sigma_{\beta+1}^{(0)}}{{ }_{t-1} \sigma_{\beta}^{(0)}} .
\end{gathered}
$$

The propagation laws of the normalized second-order moments $\langle\hat{\rho}\rangle,\langle\hat{\tau}\rangle$, and $\langle\hat{\kappa}\rangle$ through an $\mathrm{ABCD}$ system can 
be obtained applying the known theory of the phase-space distributions [15-21], we have

$$
\left[\begin{array}{c}
\langle\hat{\rho}\rangle \\
\langle\hat{\tau}\rangle \\
\langle\hat{\kappa}\rangle
\end{array}\right]=\left[\begin{array}{ccc}
A^{2} & 2 A B & B^{2} \\
A C & A D+B C & B D \\
C^{2} & 2 C D & D^{2}
\end{array}\right]\left[\begin{array}{c}
\langle\hat{\rho}\rangle_{0} \\
\langle\hat{\tau}\rangle_{0} \\
\langle\hat{\kappa}\rangle_{0}
\end{array}\right] .
$$

Equation (39) permits the evaluation of the propagation of the second-order moments of a Cartesian beam through a paraxial ABCD system once their initial values are known.

In a similar way, the propagation rules through the $\mathrm{ABCD}$ system for the fourth-order moments of a Cartesian beam are found to be $[18,20]$

$$
\mathbf{W}=\left[\begin{array}{ccc}
A^{4} & 4 A^{3} B & 6 A^{2} B^{2} \\
A^{3} C & A^{2}(A D+3 B C) & 3 A B(A D+B C) \\
A^{2} C^{2} & 2 A C(A D+B C) & A^{2} D^{2}+4 A B C D+B^{2} C^{2} \\
A C^{3} & C^{2}(3 A D+B C) & 3 C D(A D+B C) \\
C^{4} & 4 C^{3} D & 6 C^{2} D^{2} \\
4 A B^{3} & B^{4} \\
B^{2}(3 A D+B C) & B^{3} D \\
2 A B D(A D+B C) & B^{2} D^{2} \\
D^{2}(A D+3 B C) & B D^{3} \\
4 C D^{3} & D^{4}
\end{array}\right] \mathbf{W}_{0},
$$

where $\mathbf{W}$ denotes the vector of fourth-order moments

$$
\mathbf{W} \equiv\left[\begin{array}{c}
\left\langle\hat{\rho}^{2}\right\rangle \\
\langle\overline{\rho \tau}\rangle \\
\langle\hat{\epsilon}\rangle \\
\langle\overline{\tau \kappa}\rangle \\
\left\langle\hat{\kappa}^{2}\right\rangle
\end{array}\right], \quad \hat{\epsilon} \equiv \frac{\overline{\rho \kappa}+2 \hat{\tau}^{2}}{3}
$$

with the expectation values defined as

$$
\begin{aligned}
\langle\overline{\rho \tau}\rangle & \equiv\langle\hat{\rho} \hat{\tau}+\hat{\tau} \hat{\rho}\rangle / 2, \\
\langle\overline{\rho \kappa}\rangle & \equiv\langle\hat{\rho} \hat{\kappa}+\hat{\kappa} \hat{\rho}\rangle / 2, \\
\langle\overline{\tau \kappa}\rangle & \equiv\langle\hat{\tau} \hat{\kappa}+\hat{\kappa} \hat{\tau}\rangle / 2 .
\end{aligned}
$$

Like the second-order moments, the explicit expressions for $\mathbf{W}_{0}$ in terms of the parameters of the Cartesian beam can be straightforwardly obtained from (40) to (43) of [1] by making the replacement $m \rightarrow(t-1)$.

\subsection{Invariants depending on the beam moments}

The identification of quantities, named invariants, that are conserved upon propagation is of importance because they can be understood as intrinsic characteristic parameters of the beam [20]. In this subsection we identify some basic invariants of Cartesian beams that come from combinations of the lowerorder moments discussed so far.

(i) The simplest invariant is naturally the beam power $\sigma^{0}(18)$.

(ii) In terms of the second-order moments (39), we have the following invariant:

$$
\langle\hat{\rho}\rangle\langle\hat{\kappa}\rangle-\langle\hat{\tau}\rangle^{2}=\mathrm{cons},
$$

that, as we will see later, is directly related to the so-called $M^{2}$ quality factor of the beam. (iii) In terms of the fourth-order moments (41), we identify the following invariants:

$$
\begin{gathered}
\langle\overline{\rho \kappa}\rangle-\left\langle\hat{\tau}^{2}\right\rangle=\text { cons } \\
\left\langle\hat{\rho}^{2}\right\rangle\left\langle\hat{\kappa}^{2}\right\rangle-4\langle\overline{\rho \kappa}\rangle\langle\overline{\tau \kappa}\rangle+3\langle\hat{\epsilon}\rangle^{2}=\text { cons. }
\end{gathered}
$$

The physical interpretation of the fourth-order invariants is currently under study by the authors.

\section{Characterization parameters}

Finally, we remark that our formalism allows to calculate relevant characterization parameters of the Cartesian beam and therefore of all its special cases mentioned in the Introduction. Particular examples of these parameters are the following:

\subsection{Factor $M^{2}$}

The known beam quality factor $M^{2}$ is given in terms of the normalized second-order moments $\langle\hat{\rho}\rangle,\langle\hat{\tau}\rangle$, and $\langle\hat{\kappa}\rangle$ according to

$$
M^{2}=k \sqrt{\langle\hat{\rho}\rangle\langle\hat{\kappa}\rangle-\langle\hat{\tau}\rangle^{2}}
$$

For a particular plane where $\langle\hat{\tau}\rangle=0$ (i.e. at the waist of the beam), the factor $M^{2}$ reduces to

$$
M^{2}=k \sqrt{\langle\hat{\rho}\rangle\langle\hat{\kappa}\rangle}=\sqrt{\frac{\sigma^{(2)} \tilde{\sigma}^{(2)}}{\sigma^{(0)} \tilde{\sigma}^{(0)}}} .
$$

Replacing the corresponding values of the intensity moments from (14) and (32) we can express the factor $M^{2}$ in terms of the Appell hypergeometric functions as follows:

$$
\begin{aligned}
M^{2}= & t\left|\frac{q_{0}}{\operatorname{Im}\left(q_{0}\right)}\right|^{t}\left\{\left[F_{2}\left(t+1 ; \beta, \beta^{*} ; t, t ; \frac{P_{0}}{S_{0}}, \frac{P_{0}^{*}}{S_{0}}\right)\right.\right. \\
& \left.\times F_{2}\left(t+1 ; \beta, \beta^{*} ; t, t ; \frac{\tilde{P}_{0}}{\tilde{S}_{0}}, \frac{\tilde{P}_{0}^{*}}{\tilde{S}_{0}}\right)\right] \\
& \times\left[F_{2}\left(t ; \beta, \beta^{*} ; t, t ; \frac{P_{0}}{S_{0}}, \frac{P_{0}^{*}}{S_{0}}\right)\right. \\
& \left.\left.\times F_{2}\left(t ; \beta, \beta^{*} ; t, t ; \frac{\tilde{P}_{0}}{\tilde{S}_{0}}, \frac{\tilde{P}_{0}^{*}}{\tilde{S}_{0}}\right)\right]^{-1}\right\}^{1 / 2},
\end{aligned}
$$

where from (4) and (17) we have

$$
\frac{P_{0}}{S_{0}}=\frac{\mathrm{i}}{2 \operatorname{Im}\left(1 / q_{0}\right)}\left(\frac{1}{p_{0}}-\frac{1}{q_{0}}\right),
$$

and from (30) and (32) we have

$$
\frac{\tilde{P}_{0}}{\tilde{S}_{0}}=\frac{\mathrm{i}\left(p_{0}-q_{0}\right)}{2 \operatorname{Im}\left(q_{0}\right)} .
$$

As a concrete example of application of (48) we calculate the $M^{2}$ factor of the generalized Hermite-Gaussian beam $\mathrm{gHG}_{N}\left(x ; q_{0}, p_{0}\right)$ whose definition is given in the appendix (see (A.11)). In figure 1 we show the behavior of $M^{2}$ of the gHG beam with $q_{0}=-\mathrm{i} z_{R}$ in the interval $p_{0}=\left[-\mathrm{i} 8 z_{R}, \mathrm{i} 8 z_{R}\right]$ for several values of the order $N=0,1,2, \ldots$. The case $N=0$ is actually the one-dimensional Gaussian beam whose factor $M^{2}=1 / 2$ does not depend on the parameters $\left(q_{0}, p_{0}\right)$. The special value $\operatorname{Im}\left(p_{0}\right) / z_{R}=1$ corresponds to the standard Hermite-Gaussian beam for which $M^{2}=N+t$. For large values of $\left|\operatorname{Im}\left(p_{0}\right) / z_{R}\right|$ the $\mathrm{gHG}$ beam tends to the elegant $\mathrm{HG}$ beam and the curves of $M^{2}$ tend to (A.10) [22]. 

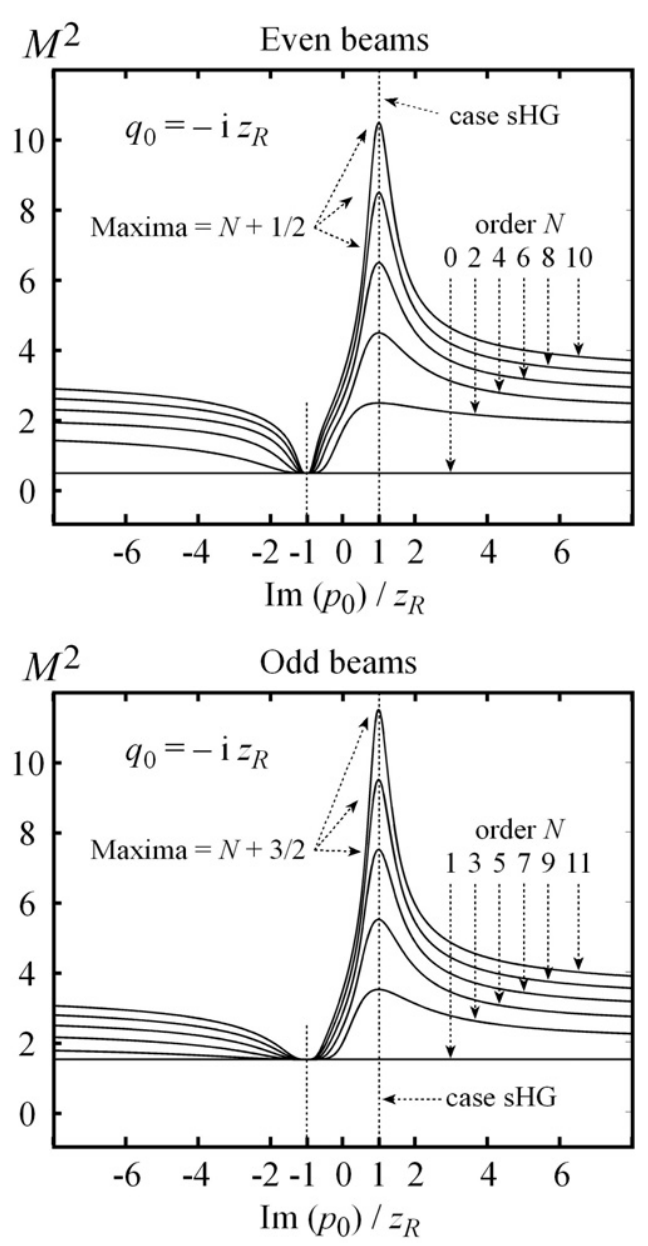

Figure 1. Factor $M^{2}$ of the generalized Hermite-Gaussian beam $\mathrm{gHG}_{N=0,1,2, \ldots}\left(x ; q_{0}, p_{0}\right)$ with $q_{0}=-\mathrm{i} z_{R}$.

\subsection{Kurtosis}

The kurtosis parameter for Cartesian beams is defined as [24]

$$
\mathcal{K}=\frac{\left\langle\hat{\rho}^{2}\right\rangle}{\langle\hat{\rho}\rangle^{2}}=\frac{\left\langle x^{4}\right\rangle}{\left\langle x^{2}\right\rangle^{2}}=\frac{\left(\sigma^{(4)} / \sigma^{(0)}\right)}{\left(\sigma^{(2)} / \sigma^{(0)}\right)^{2}}=\frac{\sigma^{(0)} \sigma^{(4)}}{\left[\sigma^{(2)}\right]^{2}},
$$

and describes the degree of flatness (or sharpness) of the beam intensity distribution. Replacing the corresponding values of the intensity moments from (14) we obtain after some straightforward simplifications the general expression of $\mathcal{K}$ in terms of the Appell hypergeometric functions, namely

$$
\begin{aligned}
\mathcal{K}= & \left(\frac{t+1}{t}\right) \\
& \times \frac{F_{2}\left(t ; \beta, \beta^{*} ; t, t ; \frac{P}{S}, \frac{P^{*}}{S}\right) F_{2}\left(t+2 ; \beta, \beta^{*} ; t, t ; \frac{P}{S}, \frac{P^{*}}{S}\right)}{\left[F_{2}\left(t+1 ; \beta, \beta^{*} ; t, t ; \frac{P}{S}, \frac{P^{*}}{S}\right)\right]^{2}},
\end{aligned}
$$

where $P$ and $S$ depend on propagation according to (4) and (17), respectively.

\subsection{The embedded Gaussian beam}

Having calculated the $M^{2}$ parameter of a Cartesian beam, one can envision an 'embedded' Gaussian beam with a generalized complex radius of curvature $Q$ defined as [26]

$$
\frac{1}{Q} \equiv \frac{\langle\hat{\tau}\rangle}{\langle\hat{\rho}\rangle}+\mathrm{i} \frac{M^{2} / k}{\langle\hat{\rho}\rangle} .
$$

Using (39) it can be demonstrated that the transformation of $Q$ undergone when passing through the $\mathrm{ABCD}$ system is the conventional bilinear transformation

$$
Q=\frac{A Q_{0}+B}{C Q_{0}+D} \text {. }
$$

By rewriting $1 / Q=1 / \mathcal{R}+i 2 / k w^{2}$, we determine the equivalent beam radius $w=\left(2\langle\hat{\rho}\rangle / M^{2}\right)^{1 / 2}$, and the radius of curvature $\mathcal{R}=\langle\hat{\rho}\rangle /\langle\hat{\tau}\rangle$ of the spherical wavefront of the embedded Gaussian.

\section{Conclusions}

We have demonstrated in this work that closed-form expressions for the overlap and the $2 v$-order intensity moments of Cartesian beams can be written in terms of the Appell hypergeometric function $F_{2}$. These expressions allow one to calculate relevant beam parameters such as the higher-order moments, the normalizations, the $M^{2}$ factors, the kurtosis parameters, and the expansion coefficients between all the relevant special cases of Cartesian beams included in table A.1. It was also possible to determine the explicit expressions for the second- and fourth-order moments of the Cartesian beam in terms of its parameters at a given initial plane of the ABCD system, and with these it was possible to calculate its propagation thorough systems.

\section{Acknowledgments}

We acknowledge support from Consejo Nacional de Ciencia y Tecnología (grant 82407) and from the Tecnológico de Monterrey (grant CAT-141).

\section{Appendix. Special cases of the Cartesian beam}

For reference purposes, in table A.1 we show a list of known special cases of the Cartesian beam and the corresponding values of the beam parameters $\left(\beta, q_{0}, p_{0}\right)$ at the initial plane $z=0$. Intensity and phase patterns at the planes $z=0$ and $z=1.5 z_{R}$ and along the plane $\left(y, 0 \leqslant z \leqslant 1.5 z_{R}\right)$ for several special cases of the Cartesian beam are shown in figure A.1. The two-dimensional beams are constructed with the product

$$
{ }_{t_{1}} U_{\beta_{x}}\left(x ; q_{x}, p_{x}\right){ }_{t_{2}} U_{\beta_{y}}\left(y ; q_{y}, p_{y}\right) .
$$

The explicit expressions of the beams, the powers ${ }_{t} \sigma^{0}$, and the $M^{2}$ factors for some of the special cases are given below. In all cases, the expressions are obtained replacing the values included in table A.1 into (2), (18), and (46). The corresponding normalization constant can be calculated with $\left({ }_{t} \sigma^{0}\right)^{-1 / 2}$. The parity parameter is given by

$$
t \equiv \begin{cases}1 / 2, & \text { for even (e) beams } \\ 3 / 2, & \text { for odd (o) beams. }\end{cases}
$$






Figure A.1. Intensity and phase patterns at the planes $z=0$ and $z=1.5 z_{R}$ and along the plane $\left(y, 0 \leqslant z \leqslant 1.5 z_{R}\right)$ for several special cases of the two-dimensional Cartesian beam ${ }_{t_{1}} U_{\beta_{x}}\left(x ; q_{x}, p_{x}\right)_{t_{2}} U_{\beta_{y}}\left(y ; q_{y}, p_{y}\right)$. In these examples we have assumed $q_{0 x}=q_{0 y}=q_{0}$ and $p_{0 x}=p_{0 y}=p_{0}$.

(This figure is in colour only in the electronic version)

Table A.1. Special cases of the Cartesian beam ${ }_{t} U_{\beta}\left(x, q_{0}, p_{0}\right)$.

\begin{tabular}{lrlll}
\hline Special cases & $\beta$ & $q_{0}$ & $p_{0}$ & Comment \\
\hline Gaussian beam [4] & 0 & $q_{0}$ & - & Obtained with $U_{\beta}$ \\
Standard $\mathrm{HG}_{N}[23]$ & $-n$ & $q_{0}$ & $q_{0}^{*}$ & $N=2 n+(t-1 / 2)$ \\
Elegant $\mathrm{HG}_{N}[23]$ & $-n$ & $q_{0}$ & $\infty$ & $N=2 n+(t-1 / 2)$ \\
Generalized $\mathrm{HG}_{N}[4]$ & $-n$ & $q_{0}$ & $p_{0}$ & $N=2 n+(t-1 / 2)$ \\
Cosine-Gaussian [5] & $-n$ & $q_{0}$ & $\left(q_{0}^{-1}-\mathrm{i} k_{t}^{2} / 2 n k\right)^{-1}$ & $\lim _{n \rightarrow \infty} U \propto \cos \left(k_{t} r\right) \exp \left(\mathrm{i} k x^{2} / 2 q_{0}\right)$ \\
Fractional eHG $\mathrm{H}_{\eta}[8]$ & $-\eta$ & $q_{0}$ & $\infty$ & See derivation below \\
Lorentz beam [6, 7] & -1 & $\infty$ & $p_{0}$ & See derivation below \\
Airy beam [9, 10] & $-n$ & $-\mathrm{i} k w^{2} / 2$ & $\mathrm{i} k w^{2} / 2$ & See derivation below \\
1D parabolic-Gaussian [3] & $\beta$ & $-p_{0}^{*}$ & $\left(k w_{0}^{2} / 2\right) /(h+\mathrm{i})$ & $\left\{w_{0}, h\right\} \in \mathbb{R}$ \\
\hline
\end{tabular}




\section{Standard Hermite-Gaussian beam $\mathrm{sHG}_{N}[4]$}

The standard Hermite-Gaussian beam $\mathrm{sHG}_{N=2 n+(t-1 / 2)}\left(x ; q_{0}\right)$, ( $n=0,1,2, \ldots)$ is obtained from (2) by replacing the parameters $\beta=-n$ and $p_{0}=q_{0}^{*}$. The propagation through an ABCD system is given by

$\operatorname{sHG}_{N}(x, z)=\zeta_{t} \frac{1}{(-1)^{n} 2^{N}} \frac{\Gamma(t)}{\Gamma(n+t)} H_{N}(\sqrt{P} x) \exp \left(\frac{\mathrm{i} k}{2 q} x^{2}\right)$,

where $P$ and $q$ are given by (4) and (5), respectively, $H_{N}$ is the Hermite polynomial of degree $N$, and

$$
\zeta_{t}=\frac{\left(A+B / q_{0}^{*}\right)^{N / 2}}{\left(A+B / q_{0}\right)^{(N+1) / 2}} .
$$

The power and $M^{2}$ factor are

$$
\begin{gathered}
{ }_{t} \sigma^{(0)}=\frac{1}{\left|P_{0}\right|^{1 / 2}} \frac{[\Gamma(t)]^{2} \Gamma(n+1)}{\Gamma(n+t)}, \\
M^{2}=2 n+t=N+1 / 2 .
\end{gathered}
$$

\section{Elegant Hermite-Gaussian beam eHG $\mathrm{eH}_{N}[4,23]$}

The elegant Hermite-Gaussian beams $\mathrm{eHG}_{N=2 n+(t-1 / 2)}\left(x ; q_{0}\right)$, $(n=0,1,2, \ldots)$ is obtained from (2) by replacing the parameters $\beta=-n$ and $p_{0}=\infty$. The propagation through an $\mathrm{ABCD}$ system is given by

$$
\mathrm{eHG}_{N}(x)=\zeta_{t} \frac{1}{(-1)^{n} 2^{N}} \frac{\Gamma(t)}{\Gamma(n+t)} H_{N}(\sqrt{P} x) \exp \left(\frac{\mathrm{i} k}{2 q} x^{2}\right)
$$

where $P$ and $q$ are given by (4) and (5), respectively, and

$$
\zeta_{t}=A^{N / 2}\left(A+B / q_{0}\right)^{-(N+1) / 2}
$$

The power and $M^{2}$ factor are

$$
\begin{gathered}
{ }_{t} \sigma^{(0)}=\sqrt{\frac{2}{k}} \frac{\Gamma(t)^{2} \Gamma(2 n+t)}{[\Gamma(n+t)]^{2}}\left|q_{0}\right|^{1 / 2}\left|\frac{P_{0}}{S_{0}}\right|^{2 n+t}, \\
M^{2}=\sqrt{\frac{(4 N-1)(2 N+1)}{8 N-4}} .
\end{gathered}
$$

\section{Generalized Hermite-Gaussian beam $g H G_{N}$ [23]}

The generalized Hermite-Gaussian beam $\mathrm{gHG}_{N=2 n+(t-1 / 2)}(x$; $\left.q_{0}, p_{0}\right),(n=0,1,2, \ldots)$ is obtained from (2) by replacing the parameter $\beta=-n$. The propagation through an ABCD system is given by

$$
\mathrm{gHG}_{N}(x)=\frac{\zeta_{t}}{(-1)^{n} 2^{N}} \frac{\Gamma(t)}{\Gamma(n+t)} H_{N}(\sqrt{P} x) \exp \left(\frac{\mathrm{i} k}{2 q} x^{2}\right),
$$

where $P$ and $q$ are given by (4) and (5), respectively, and

$$
\zeta_{t}=\frac{\left(A+B / p_{0}\right)^{N / 2}}{\left(A+B / q_{0}\right)^{(N+1) / 2}} .
$$

The power is

$$
\begin{aligned}
{ }_{t} \sigma^{(0)} & =\frac{\Gamma(t)}{S_{0}}\left|P_{0}^{1 / 2}\left(\frac{P_{0}}{S_{0}}\right)^{t-1}\left(\frac{S_{0}-P_{0}}{S_{0}}\right)^{2 n}\right| \\
& \times F\left(-n,-n ; t ;\left|\frac{P_{0}}{S_{0}-P_{0}}\right|^{2}\right) .
\end{aligned}
$$

The $M^{2}$ factor of the gHG beam is given by (48) with $\beta=-n$.

\section{Fractional-order elegant Hermite-Gaussian beams fr-eHG ${ }_{\eta}[8]$}

The fractional-order elegant Hermite-Gaussian beam of order $\eta \in \mathbb{R}$ corresponds to the $\eta$ th fractional derivative of the Gaussian function and are obtained by the superposition

$$
\begin{gathered}
\operatorname{fr}_{-} \mathrm{eHG}_{\eta}\left(x, q_{0}, p_{0}\right) \equiv \cos \left(\frac{\pi \eta}{2}\right) \Gamma\left(\frac{1+\eta}{2}\right){ }_{e} U_{-\eta}\left(x ; q_{0}, \infty\right) \\
-\sin \left(\frac{\pi \eta}{2}\right) \eta \Gamma\left(\frac{\eta}{2}\right){ }_{o} U_{-\eta}\left(x ; q_{0}, \infty\right) .
\end{gathered}
$$

\section{Cosine-Gaussian and Cosh-Gaussian beams CG [5]}

At $z=0$, the cosine-Gauss beam is obtained from the Cartesian beam ${ }_{t} U_{\beta}\left(x, p_{0}, q_{0}\right)$ in the following limit:

$$
\lim _{n \rightarrow \infty} U_{-n}\left(x ; q_{0}, \frac{1}{q_{0}^{-1}-\mathrm{i} k_{t}^{2} / 2 n k}\right) \propto \cos \left(k_{t} r\right) \exp \left(\frac{\mathrm{i} k x^{2}}{2 q_{0}}\right) .
$$

The propagation through an $\mathrm{ABCD}$ system is given by

$$
\mathrm{CG}(x)=\frac{\Gamma(n+1)}{\sqrt{n} \Gamma(n+1 / 2)} \zeta \exp \left(\frac{\mathrm{i} k x^{2}}{2 q}\right) \cos \left(\zeta^{2} k_{t} x\right)
$$

where $\zeta=1 / \sqrt{A+B / q_{0}}$.

The power and $M^{2}$ factor are

$$
\begin{gathered}
\sigma^{(0)}=\left[\frac{\Gamma(t) \Gamma(n+1) n^{(t-1) / 2}}{\Gamma(n+t)}\right]^{2} \frac{\left|P_{0}\right|^{1 / 2}}{S_{0}} \frac{\mathrm{I}_{t-1}(\alpha)}{\exp (\alpha)}, \\
M^{2}=\sqrt{\left[t+\alpha \frac{I_{t}(\alpha)}{I_{t-1}(\alpha)}\right]^{2}-\alpha^{2}} .
\end{gathered}
$$

where $\mathrm{I}_{\kappa}$ is the modified Bessel function of the first kind and $\alpha \equiv k_{t}^{2} /\left(2 S_{0}\right)=k_{t}^{2} /\left[2 k \operatorname{Im}\left(1 / q_{0}\right)\right]$ for $k_{t} \in \mathbb{R}$.

Lorentz, beam $L B[6,7]$

We first define the beam

$$
U^{ \pm} \equiv{ }_{e} U_{1 / 2}\left(w \pm i x, \infty, p_{0}\right)-\frac{2}{\sqrt{\pi}}{ }_{o} U_{1}\left(w \pm \mathrm{i} x, \infty, p_{0}\right),
$$

where $w$ is a parameter and

$$
\begin{gathered}
{ }_{e} U_{1 / 2}(w \pm \mathrm{i} x)=\frac{1}{\sqrt{A+B / p_{0}}}{ }_{1} F_{1}\left(\frac{1}{2}, \frac{1}{2}, P(w \pm \mathrm{i} x)^{2}\right), \\
{ }_{o} U_{1}(w \pm \mathrm{i} x)=\frac{\sqrt{P(w \pm \mathrm{i} x)^{2}}}{\sqrt{A+B / p_{0}}}{ }_{1} F_{1}\left(1, \frac{3}{2}, P(w \pm \mathrm{i} x)^{2}\right) .
\end{gathered}
$$


The Lorentz beam is obtained by the superposition $\mathrm{LB}(x)=U^{+}+U^{-}$. Replacing and using the identity

$\exp \left(z^{2}\right)[1-\operatorname{erf}(z)]={ }_{1} F_{1}\left(\frac{1}{2}, \frac{1}{2}, z^{2}\right)-\frac{2 z}{\sqrt{\pi}}{ }_{1} F_{1}\left(1, \frac{3}{2}, z^{2}\right)$,

where erf is the error function, we obtain

$$
\begin{gathered}
\mathrm{LB}(x)=\frac{\exp \left[P(w+\mathrm{i} x)^{2}\right]}{\sqrt{A+B / p_{0}}}\left[1-\operatorname{erf}\left(\sqrt{P(w+\mathrm{i} x)^{2}}\right)\right] \\
+\frac{\exp \left[P(w-\mathrm{i} x)^{2}\right]}{\sqrt{A+B / p_{0}}}\left[1-\operatorname{erf}\left(\sqrt{P(w-\mathrm{i} x)^{2}}\right)\right],
\end{gathered}
$$

which is the general case of (15) in [6].

At the initial plane $z=0$, for the special case when $w=w_{0} \in \mathbb{R}$ and $p_{0}=-\mathrm{i} k w_{0}^{2} / 2$, we obtain

$$
\begin{gathered}
\operatorname{LB}(x)=\frac{2}{\sqrt{\pi}} \frac{1}{1+\left(x / w_{0}\right)^{2}}, \\
\sigma^{(0)}=2 w_{0}, \\
M^{2}=1 / \sqrt{2} .
\end{gathered}
$$

which is the case reported in $[6,7]$ where the distribution at $z=0$ reduces to a Lorentzian function.

\section{Airy beam [9, 10]}

The Airy beam $A i(x / \kappa)$ is a special case of the Cartesian beam. The relation of the Airy beam to the standard HermiteGaussian beam is given by

$$
A i(x / \kappa)=\lim _{n \rightarrow \infty} \operatorname{sHG}_{n}\left(x+\Delta_{n}, w_{n}\right),
$$

where

$$
\begin{gathered}
w_{n} \equiv 2 \kappa n^{1 / 6}, \quad \Delta_{n}=2 \kappa n^{2 / 3} \\
\operatorname{sHG}_{n}(x, w) \propto H_{n}\left(\frac{\sqrt{2} x}{w}\right) \exp \left(-\frac{x^{2}}{w^{2}}\right) .
\end{gathered}
$$

One-dimensional parabolic-Gaussian beam ${ }_{t} \Theta_{\beta}(x)[3$, equation (16)]

The possibility of choosing arbitrary complex values for the parameters $\left(\beta, q_{0}, p_{0}\right)$ allows us to obtain new meaningful beam structures from the general expression of the Cartesian beam (2). For example, the combination of values in the last row of table A.1 produces new solutions ${ }_{t} \Theta_{\beta}(x)$ whose transverse field at $z=0$ is given by [3]

$$
{ }_{t} \Theta_{\beta}(x)=\exp \left(-\frac{x^{2}}{w_{0}^{2}}\right){ }_{t} \mathrm{P}_{\alpha}\left(2 \sqrt{h} \frac{x}{w_{0}}\right),
$$

where ${ }_{t} \mathrm{P}_{\alpha}$ is the parabolic cylinder function of parity $t=\{\mathrm{e}, \mathrm{o}\}$ (section 19.16 of [25]) and parameter $\alpha=\mathrm{i}(2 \beta-t)$.

The power and $M^{2}$ factor of the beam ${ }_{t} \Theta_{\beta}(x)$ are

$$
\begin{gathered}
{ }_{t} \sigma_{\beta}^{(0)}=\Gamma(t) \frac{w_{0}}{\sqrt{2}}\left|\frac{h^{t-1 / 2}}{(1-\mathrm{i} h)^{2 \beta}}\right| F\left(\beta, \beta^{*} ; t ; \frac{h^{2}}{1+h^{2}}\right), \\
M^{2}=\sqrt{\frac{u^{2}-|\alpha|^{2}}{1+h^{-2}}},
\end{gathered}
$$

where

$$
u \equiv \frac{t}{h}+\frac{2|\beta|^{2}}{t^{2}} \frac{{ }_{t} \sigma_{\beta+1}^{(0)}}{{ }_{t-1} \sigma_{\beta}^{(0)}} \text {. }
$$

\section{References}

[1] Bandres M A, Lopez-Mago D and Gutiérrez-Vega J C 2010 Higher-order moments and overlaps of rotationally symmetric beams J. Opt. 12015706

[2] Bandres M A and Gutiérrez-Vega J C 2008 Circular beams Opt. Lett. 33 177-9

[3] Bandres M A and Gutiérrez-Vega J C 2007 Cartesian beams Opt. Lett. 32 3459-61

[4] Siegman A E 1986 Lasers (Mill Valley, CA: University Science Books)

[5] Gutiérrez-Vega J C and Bandres M A 2005 Helmholtz-Gauss waves J. Opt. Soc. Am. A 22 289-98

[6] El Gawhary O and Severini S 2006 Lorentz beams and symmetry properties in paraxial optics J. Opt. A: Pure Appl. Opt. 8 409-14

[7] El Gawhary O and Severini S 2006 Lorentz beams as a basis for a new class of rectangularly symmetric optical fields $O p t$. Commun. 269 274-84

[8] Gutiérrez-Vega J C 2007 Fractionalization of optical beams: I. Planar analysis Opt. Lett. 32 1521-3

[9] Siviloglou G A and Christodoulides D N 2007 Accelerating finite energy Airy beams Opt. Lett. 32 979-81

[10] Bandres M A and Gutiérrez-Vega J C 2007 Airy-Gauss beams and their transformation by paraxial optical systems $O p t$. Express 15 16719-28

[11] Torre A 2008 A note on the general solution of the paraxialwave equation: a Lie algebra view J. Opt. A: Pure Appl. Opt. 10055006

[12] Torre A 2010 Linear and quadratic exponential modulation of the solutions of the paraxial wave equation $J$. Opt. 12035701

[13] Saad N and Hall R L 2003 Integrals containing confluent hypergeometric functions with applications to perturbed singular potentials J. Phys. A: Math. Gen. 36 7771-88

[14] Gradshteyn I S and Ryzhik I M 2007 Table of Integrals, Series, and Products ed A Jeffrey and D Zwillinger (Amsterdam: Academic)

[15] Bastiaans M J 1989 Propagation law for the second-order moments of the wigner distribution function in first-order optical systems Optik 82 173-81

[16] Bastiaans M J 1991 Second-order moments of the Wigner distribution function in first-order optical systems Optik $\mathbf{8 8}$ $163-8$

[17] Onciul D 1993 Invariance properties of general astigmatic beams through first-order optical systems J. Opt. Soc. Am. A 10 295-9

[18] Dragoman D 1994 Higher-order moments of the Wigner distributions function in first-order optical systems $J$. Opt. Soc. Am. A 11 2643-6

[19] Belanger P A, Champagne Y and Pare C 1994 Beam propagation factor of diffracted laser beams Opt. Commun. $105233-42$

[20] Dodonov V V and Man'ko O V 2000 Universal invariants inquantum mechanics and physics of optical and particle beams J. Russian Laser Res. 21 438-64

[21] Torre A 2005 Linear Ray and Wave Optics in Phase Space (Amsterdam: Elsevier)

[22] Saghafi S and Sheppard C J R 1998 The beam propagation factor for higher order Gaussian beams Opt. Commun. $153207-10$

[23] Saghafi S, Sheppard C J R and Piper J A 2001 Characterising elegant and Hermite-Gaussian modes Opt. Commun. 191 173-9

[24] Martínez-Herrero R, Piquero G and Mejías P M 1995 On the propagation of the kurtosis parameter of general beams $O p t$. Commun. 115 225-32

[25] Abramowitz M and Stegun I A 1964 Handbook of Mathematical Functions (New York: Dover)

[26] Siegman A E 1991 Defining the effective radius of curvature for a nonideal optical beam IEEE J. Quantum Electron. 27 1146-8 